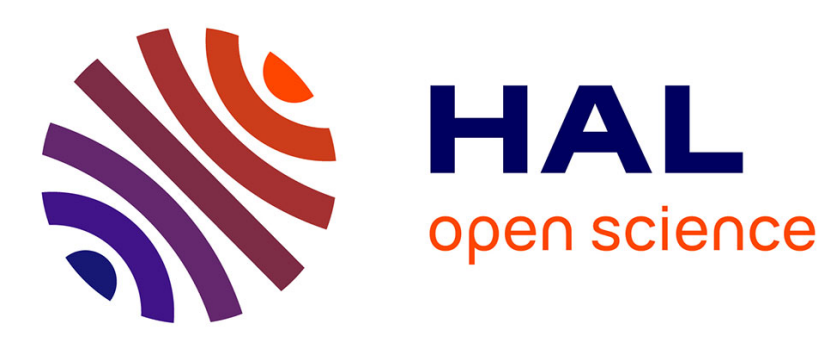

\title{
Cournot duopoly and insider trading with two insiders
}

Wassim Daher, Leonard J Mirman

\section{To cite this version:}

Wassim Daher, Leonard J Mirman. Cournot duopoly and insider trading with two insiders. 2004. halshs-03331519

\section{HAL Id: halshs-03331519 \\ https://shs.hal.science/halshs-03331519}

Submitted on 1 Sep 2021

HAL is a multi-disciplinary open access archive for the deposit and dissemination of scientific research documents, whether they are published or not. The documents may come from teaching and research institutions in France or abroad, or from public or private research centers.
L'archive ouverte pluridisciplinaire HAL, est destinée au dépôt et à la diffusion de documents scientifiques de niveau recherche, publiés ou non, émanant des établissements d'enseignement et de recherche français ou étrangers, des laboratoires publics ou privés. 

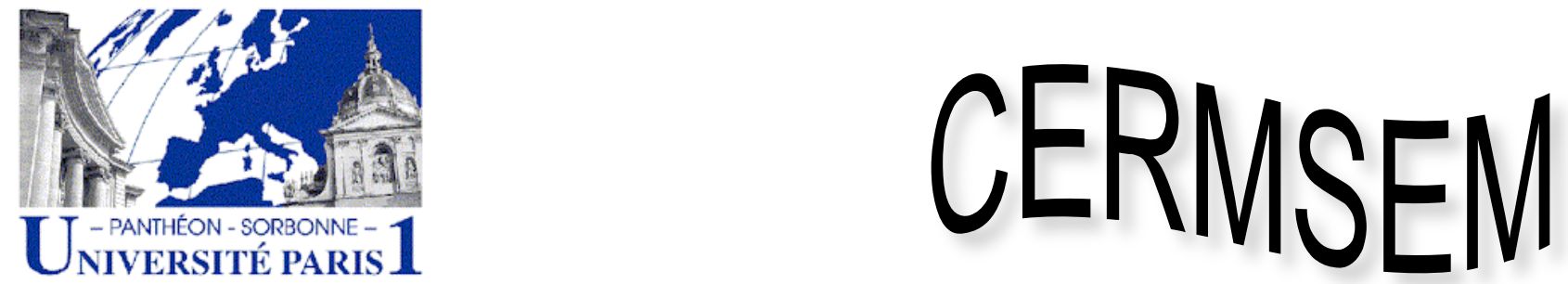

UMR CNRS 8095

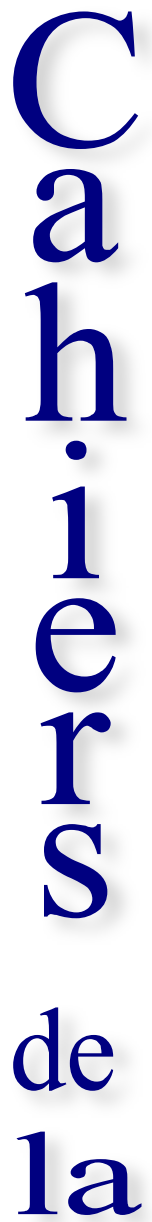

Cournot Duopoly and Insider Trading with two Insiders

Wassim DAHER

Leonard J. MIRMAN

2004.77
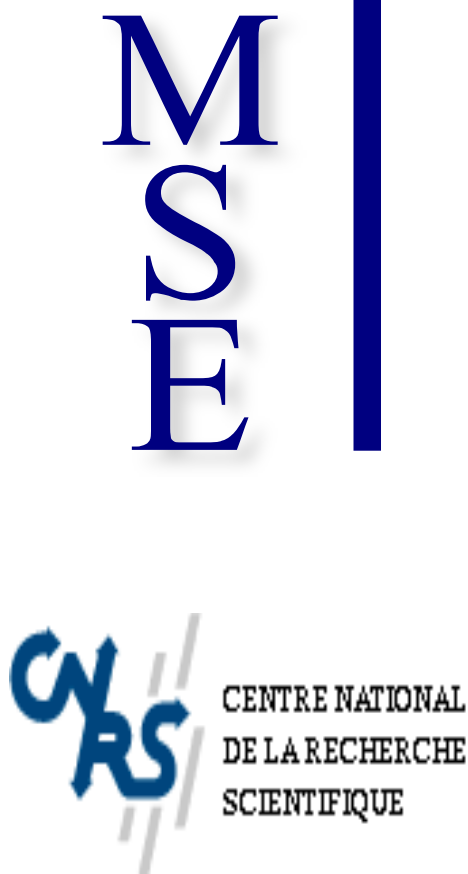

Maison des Sciences Économiques, 106-112 boulevard de L'Hôpital, 75647 Paris Cedex 13 


\title{
COURNOT DUOPOLY AND INSIDER TRADING WITH TWO INSIDERS
}

\author{
WASSIM DAHER AND LEONARD J. MIRMAN
}

\begin{abstract}
In this paper, we study a version of the static Jain-Mirman (2002) model in which competition in the real sector is introduced. In this paper, we add competition in the stock sector to the Jain-Mirman(2002) paper. We show that the linear equilibrium structure is affected by this competition in the financial sector. Specifically, we show that the stock price set by the market maker reveals more information and that the behavior of the profits of the manager depends on the parameters of the model. Moreover, we prove that the level of output produced by the manager is less than in Jain-Mirman (2002). Finally, we also study the case in which the market maker receives only one signal and analyze the comparative statics of this model when the market maker receives either one or two signals.
\end{abstract}

Keywords: Insider Trading, Stock prices, Correlated signals, Kyle model

JEL Classification: G14, D82

\section{INTRODUCTION}

Economics environments that study the effect of financial decisions of the firm should also include the effect of these decisions on the real aspects of the firm since these are inextricably intertwined. Ignoring either aspect of a firm that depends on external financing like a public firm misses a significant constraint on the ability to perform in the real sector. For example the work of Kyle (1985) in studying insider trading does not account for the effect of the insiders trades on the real part of the firm. Although many insights about the trades of the insider and the informational content of these trades are possible in this model, the impact on the real sector misses an important ingredient of the effect of insider trading on the firm. This point is also apparent when studying the effect of competition, with more than one trader, on the financial side of the firm, as was studied by Tighe (1989). In both of these cases the influence of the insider on the profitability of the firm is ignored. Thus, the trades and the informational content of decisions are ignored.

This issue was addressed in several papers by Jain and Mirman, in which the real and the financial aspect of insider trading were inextricably related. In JM (2000) two basic changes to the Kyle model are made. The first is that the real sector of the economy is added, so that the insider can make real decisions of the firm as well as financial decisions. Second the market maker can see two signals, a real signal and the order flow signal. The 
model is designed to yield a linear equilibrium so that the comparative statics are both simple and workable. In JM there is no competition either in the financial sector or the real sector, competition in the real sector was added to this model in JMC (2002). In these models the results of Kyle is shown to be altered by the insider, the manager of the firm, to influence the real output and, thus, the profitability of the firm. This connection also had a profound effect on the information that is revealed by insider trading. Indeed, it is shown in JM that the amount of information incorporated in the stock price is a function of the variables of the model rather than a constant, as in Kyle. Moreover, the amount of information in JM is greater than in Kyle and the profits of the manager are also less than Kyle. Adding a competitive firm, as in JMC, does not change the revelation of information relative to JM but, due to the Cournot structure in the real sector, the profits of the manager, as well as the compensation received by the manager, are less than in JM. In Daher and Mirman (2004), competition in the financial sector was added to JM in the form of a second inside trader. It is shown that this competition on the financial side of the firm, although similar to the effect of an additional trader in the Kyle model, has a profound effect on the results of JM. Indeed, the profits of the firm, as well as the trading strategies, are altered in an interesting and significant way. In particular, it is shown in DM that adding another informed trader in the stock market increases the amount of information revealed by the stock price relative to JM. In contrast, the profits of the manager are not always less than in JM but depend on the variances of the exogenous variables. Moreover, the trading level of the owner in DM resembles the trading level in Tighe and the trading level of the manager resembles the trading level of the manager in JM. These results are due to the presence of the compensation scheme received by the manager in JM and DM. In this paper we combine the effect of the JMC (competition among firms) and DM (competition among insiders) and study the effect of competition in both the real and the financial sector, simultaneously. This adds richness and depth to the model and allows us to study the relationship between the real and financial sectors. This was the object of JM when the real sector was introduced in the insider trading model of Kyle. However, this paper has extended the model in JM by adding competition both the real as well as the financial sector, allowing us to study how the different market structures depend on each other. Indeed, we show that the decisions made in the financial markets depend on the industrial structure in the real market, i.e., the results depend on the type of competition in the real sector. We also show that the decisions in the real market are influenced by the competition in the financial markets. We compare the equilibrium of this paper to JMC and DM. First, we show that adding a second firm to DM, i.e., adding Cournot competition, decreases the value of the firm and, thus, the coefficients of the stock price set by the market maker, decrease. Moreover, the amount of information revealed by the stock price remains unchanged by the competition in the real sector. Note that the comparative statics between this paper and DM is similar to the one between JMC and JM. Second, the relationship between JMC and this paper is similar to the relationship between DM and JM. In fact, relative to JMC, we show that adding a second informed trader increases the amount of information incorporated in the stock price. We also show that the profits 
of the manager depend on the variances of the exogenous variables. Next, we show that the intercept coefficient of the stock price is affected by competition in the financial sector. Moreover, the profits of the manager are also affected by the financial competition. Indeed, compared to JM, one would expect that the profits of the manager are less than in JM, due to Cournot competition. But this is not the case since the profits of the firm depend on the number insiders trading. Hence, we show that the profits, relative to JM, sometimes increase and sometimes decrease, depending on the variances of the exogenous variables. Finally, the coefficient of the real signal is affected by competition in both sectors. Indeed, going from JM to DM or from JM to JMC, the real signal coefficient decreases. Thus, going from JM to this paper mu1 decreases as in JMC or DM. In contrast, the total order flow signal is affected by competition in the financial sector. In particular, relative to JM, the order flow coefficient sometimes decreases and sometimes increase similar to the case going from JM to DM. Indeed the effect of competition in the real sector on the stock price coefficients depends crucially on the nature of competition in the financial sector.

\section{The Model}

We consider in this paper a version of the Jain-Mirman model (henceforth JMC, 2002). ${ }^{1}$ The economy consists of one real good and one financial asset. In the real market, we consider a duopoly producing the real good at no cost. The inverse demand function facing this industry is stochastic and linear, i.e.,

$$
q^{\prime}=(a-b Y) \tilde{z}, \quad a, b>0 .
$$

The prior distribution of $\tilde{z}$ is normal with mean $\bar{z}$ (assumed to be positive) and variance $\sigma_{z}^{2}, q^{\prime}$ is the real price of the output and $Y=y_{1}+y_{2}$, the aggregate quantity produced. Both firms are standard neoclassical firms with respect to the real sector. With respect to the financial sector, firm 2 is privately owned with its financial decisions ignored, while firm 1 is publicly owned. In particular, firm 1 is managed by an insider whose knowledge can be used in stock market. In the financial market, the asset is the stock of firm 1 , the value of the asset is the net profits of the firm per share. The stock of firm 1 is publicly traded in a competitive stock market. We add another insider to the JMC model, an "owner". ${ }^{2}$ The owner is assumed to have the same private information about the financial asset as the manager. The two insiders trade in the stock market based on their inside information. Before the start of trading, the random variable $\tilde{z}$ takes a realized value, denoted $z .^{3}$. Three types of agents trade. First, there are two risk-neutral rational traders: the manager and the owner of firm 1, each of whom knows $z$ with certainty. Second, there are the nonrational noise traders. They represent small investors with no information on $z$. The noise trade is assumed to be a random variable $\tilde{u}$, which is normally distributed with

\footnotetext{
${ }^{1}$ Section 3 .

${ }^{2}$ In our model, the owner is another informed agent in the firm. For example a majority shareholder or CEO, who does not have any managerial responsibilities.

${ }^{3}$ Random variables are denoted with a tilde. Realized values lack the tilde. The mean of the random variable is denoted with bar.
} 
mean zero and variance $\sigma_{u}^{2}$. Finally, there are $K(K \geq 2)$ risk-neutral market makers who act like "Bertrand" competitors. We assume, as in JMC, that the market makers observe a noisy signal from the real market, denoted by the random variable $\tilde{q}=(a-b Y)(\tilde{z}+\tilde{\varepsilon})$, where $\tilde{\varepsilon}$ is normally distributed with mean zero and variance $\sigma_{\varepsilon}^{2}$. We assume that $\tilde{z}, \tilde{u}$ and $\tilde{\varepsilon}$ are independent.

Following Kyle (1985), the trading mechanism is organized in two steps. In step one, market makers determine the pricing rule $p$, based on their a priori beliefs, where $p$ is a measurable function $p: \mathbb{R} \times \mathbb{R} \longrightarrow \mathbb{R}$. Each insider chooses a stock trade $\tilde{x}_{i}=x_{i}(\tilde{z})$ where $x_{i}: \mathbb{R} \longrightarrow \mathbb{R}$ a measurable function. In the second step, the insiders observe the value of $\tilde{z}$ and market makers observe the two signals, the real signal $\tilde{q}$ and the total order flow $\tilde{r}=x_{1}(\tilde{z})+x_{2}(\tilde{z})+\tilde{u}=x(\tilde{z})+\tilde{u}$, the combined order of the insiders and the noise traders, and set the price $\tilde{p}=p(\tilde{q}, \tilde{r})$ to clear the market. Before making his decision, each insider does not know $u, \varepsilon, r, z+\varepsilon$. Moreover, each market maker does not know $\tilde{z}$ but only knows its distribution. Also the market makers do not observe either $x_{1}, x_{2}, u$ or $\varepsilon$.

The value per share of the firm is defined to be the net profits of the firm per share. Hence, the values of firm 1 and 2 are respectively, $v^{\prime}=(a-b Y) y_{1} \tilde{z}$ and $v_{2}=(a-b Y) y_{2} \bar{z}$. Profits for each of the two rational trader are given respectively by $\Pi_{1}:=\left(v^{\prime}-B-p\right) \cdot \tilde{x_{1}}+B \tilde{x_{1}}$ and $\Pi_{2}:=\left(v^{\prime}-B-p\right) \cdot \tilde{x_{2}}$, where $B$ is the compensation received by the manager for his managerial tasks. ${ }^{4}$ When the manager receives his compensation, the value of firm 1 becomes, $\tilde{v}=v^{\prime}-B$.

This is a game of incomplete information because the market makers unlike the insiders do not know the realization of $\tilde{z}$. Hence, we seek for Bayesian Nash equilibrium. An equilibrium is a vector of five functions $\left[y_{1}(),. y_{2}(),. x_{1}(),. x_{2}(),. p(.,).\right]$ such that:

(a) Profit maximization of firm 2,

$$
\left(\left(a-b\left(y_{1}+y_{2}\right)\right) y_{2} \bar{z} \geq\left(\left(a-b\left(y_{1}+y_{2}^{\prime}\right)\right) y_{2}^{\prime} \bar{z}\right.\right.
$$

for any level of output $y_{2}^{\prime}$ produced by the firm 2 ;

(b) Profit maximization of the manager,

$$
\begin{aligned}
E\left[\left(\left(a-b\left(y_{1}\right.\right.\right.\right. & \left.\left.\left.\left.+y_{2}\right)\right) y_{1} \tilde{z}-p\left(x_{1}(\tilde{z})+x_{2}(\tilde{z})+\tilde{u}\right)\right) x_{1}(\tilde{z})\right] \\
& \geq E\left[\left(\left(a-b\left(y_{1}^{\prime}+y_{2}\right)\right) y_{1}^{\prime} \tilde{z}-p\left(x_{1}^{\prime}(\tilde{z})+x_{2}(z)+\tilde{u}\right)\right) x_{1}^{\prime}(\tilde{z})\right]
\end{aligned}
$$

for any level of output $y_{1}^{\prime}$ produced by the firm 1 and any alternative trading strategy $x_{1}^{\prime}(\tilde{z})$

(c) Profit maximization of the owner,

$$
\begin{aligned}
E\left[\left(\left(a-b\left(y_{1}\right.\right.\right.\right. & \left.\left.\left.\left.+y_{2}\right)\right) y_{1} \tilde{z}-B-p\left(x_{1}(\tilde{z})+x_{2}(\tilde{z})+\tilde{u}\right)\right) x_{2}(\tilde{z})\right] \\
& \geq E\left[\left(\left(a-b\left(y_{1}+y_{2}\right)\right) y_{1} \tilde{z}-B-p\left(x_{1}(\tilde{z})+x_{2}^{\prime}(\tilde{z})+\tilde{u}\right)\right) x_{2}^{\prime}(\tilde{z})\right]
\end{aligned}
$$

\footnotetext{
${ }^{4}$ It is shown in JMC that a compensation scheme for the manager of firm 1 is needed to ensure the existence of the linear equilibrium. This argument continues to be applicable in this paper. Indeed, we show, in the next section, that the second order condition is not always satisfied for all $z$ and then the existence of this compensation is to satisfy the second order condition for all $z$.
} 
for any alternative trading strategy $x_{2}^{\prime}(\tilde{z})$;

(d) Semi-Strong Market Efficiency: The pricing rule $p(.,$.$) satisfies,$

$$
p(\tilde{q}, \tilde{r})=E[\tilde{v} \mid \tilde{q}, \tilde{r}] .
$$

An equilibrium is linear if there exists constants $\mu_{0}, \mu_{1}, \mu_{2}$ such that,

$$
\forall q, r, p(q, r)=\mu_{0}+\mu_{1} q+\mu_{2} r .
$$

Note that conditions (2), (3) and (4) define optimal strategies of the two firms and the owner while condition (5) guarantees the zero expected profits for the market maker. Since the stock price set by the market maker is equal to the conditional expectation of the asset value given his information, we restrict our research to linear equilibrium. The normal distributions of the exogenous random variables, together with the particular expression of the demand, enable us to derive and to prove the existence of a unique linear equilibrium.

\section{Derivation of Equilibrium}

In order to derive the unique linear equilibrium, we start by solving the maximization problem of the two firms as well as the owner of firm 1. The objective of firm 2 is to produce an optimal quantity of outputs, $y_{2}($.$) , to maximize,$

$$
v_{2}=(a-b Y) y_{2} \bar{z} \text {. }
$$

The first order condition is,

$$
y_{2}=\frac{\left(a-b y_{1}\right)}{2 b} .
$$

The decision rule of the manager of firm 1 is the pair $\left(y_{1}(\tilde{z}), x_{1}(\tilde{z})\right)$.The manager expected profits are,

$$
G_{1}=E\left[\left(\left(a-b\left(y_{1}+y_{2}\right)\right) y_{1} \tilde{z}-p\left(x_{1}(\tilde{z})+x_{2}(\tilde{z})+\tilde{u}\right)\right) x_{1}(\tilde{z})\right] .
$$

The first order conditions are,

$$
x_{1}(\tilde{z})=\frac{(a-b Y)\left(y_{1}-\mu_{1}\right) \tilde{z}-\mu_{0}-\mu_{2} x_{2}(\tilde{z})}{2 \mu_{2}} \text { and } y_{1}=\frac{\left(a-b y_{2}+\mu_{1} b\right)}{2 b} .
$$

Computing (8) and (10), we get,

$$
y_{1}=\frac{\left(a+2 \mu_{1} b\right)}{3 b} \text { and } y_{2}=\frac{\left(a-\mu_{1} b\right)}{3 b} .
$$

The owner of firm 1 chooses his decision rule, $x_{2}(\tilde{z})$ to maximize his expected profits,

$$
G_{2}=E\left[\left(\left(a-b\left(y_{1}+y_{2}\right)\right) y_{1} \tilde{z}-B-p\left(x_{1}(\tilde{z})+x_{2}(\tilde{z})+\tilde{u}\right)\right) x_{2}(\tilde{z})\right]
$$

The first order condition is,

$$
x_{2}(\tilde{z})=\frac{(a-b Y)\left(y_{1}-\mu_{1}\right) \tilde{z}-\mu_{0}-B-\mu_{2} x_{2}(\tilde{z})}{2 \mu_{2}} .
$$


Computing (10) and (13), we have,

$$
x_{1}(\tilde{z})=\frac{(a-b Y)\left(y_{1}-\mu_{1}\right) \tilde{z}-\mu_{0}+B}{3 \mu_{2}} \text { and } x_{2}(\tilde{z})=\frac{(a-b Y)\left(y_{1}-\mu_{1}\right) \tilde{z}-\mu_{0}-2 B}{3 \mu_{2}} \text {. }
$$

Remark 3.1. First, note that the outputs produced by the two firms are deterministic. Hence, the trading level of each insider is an affine function of $\tilde{z}$ and thus the trading strategies of the insiders are normal random variables. Second, the two signals received by the market maker, i.e. the real signal $\tilde{q}$ and the total order flow signal $\tilde{r}$, are both normal random variables (since $\tilde{z}, \tilde{u}$ and $\tilde{\varepsilon}$ are independent). Third, since the outputs are deterministic, the value of firm 1 is also a normal random variable. Consequently, the normality of these random variables allows us to derive an explicit linear form for their conditional expected values. Hence, the stock price set by the market maker, which satisfies his zero expected profits condition, is linear. ${ }^{5}$

Lemma 1. Under competition in the real and financial sectors, the coefficients of the price function are,

(i) $\mu_{0}=\bar{v}-\mu_{1} \bar{q}-\mu_{2} \bar{r}$,

(ii) $\mu_{1}=\frac{a \sigma_{z}^{2}}{b\left(\sigma_{z}^{2}+9 \sigma_{\varepsilon}^{2}\right)}$,

(iii) $\mu_{2}=\frac{\sqrt{2} a^{2} \sigma_{\varepsilon} \sqrt{(1-k)^{3} k}}{9 b \sigma_{u}}$,

where $k=\frac{\sigma_{z}^{2}}{\left(\sigma_{z}^{2}+9 \sigma_{\varepsilon}^{2}\right)}$.

Proof: See the Appendix.

The second order conditions for the manager are,

$$
2 b x_{1}(\tilde{z}) \tilde{z}>0 \text { and } \mu_{2}>0,
$$

and the Hessian determinant is positive. For the owner, $\mu_{2}>0$. By Lemma 1, the second order condition for the owner is satisfied. In contrast, the first condition in (14) is not always satisfied since the support of the normal random variables is the entire real line. Specifically, substituting the value of $x_{1}$ in the left hand condition in (14), we find,

$$
\left[b\left(y_{1}-\mu_{1}\right)^{2}(\tilde{z}-\bar{z})+3 B\right] \tilde{z}>0 .
$$

Since the second order condition in (15) is quadratic, there exits values of $\tilde{z}$ such that the second order condition is not satisfied. Now, we present a necessary and sufficient condition for the existence of the linear equilibrium for all $\tilde{z}$.

Proposition 1. A linear equilibrium exists if and only if,

$$
B=\mu_{0}=\frac{(a-b Y)\left(y_{1}-\mu_{1}\right)}{3} \bar{z}
$$

\footnotetext{
${ }^{5}$ It should be pointed out that adding another informed trader to JMC does not change the distributions of the equilibrium outcome and thus Theorem 1, of the Appendix, applies as in JMC. Hence, the stock price set by the market maker has a linear conditional expectation form.
} 
Proof: We start by proving sufficiency. If $B=\mu_{0}$, then the expression for $x_{1}$ becomes, $x_{1}(\tilde{z})=\frac{(a-b Y)\left(y_{1}-\mu_{1}\right) \tilde{z}}{3 \mu_{2}}$. Thus, the second order condition is satisfied. Hence, a linear equilibrium exists. Next, we prove the necessity. Indeed, since there exists a linear equilibrium, the second order condition is satisfied for all $\tilde{z}$. The second order condition has the form, $c \tilde{z}^{2}+d \tilde{z}>0$. Here $c=b\left(y_{1}-\mu_{1}\right)^{2}>0$ and $d=3 B-b\left(y_{1}-\mu_{1}\right)^{2} \bar{z}$. So, we must have $d=0$ to satisfy the second order condition and thus the result is proved.

Remark 3.2. Note that if $\tilde{z}$ is centered, i.e. $\bar{z}=0$, then firm 2's profits are identically zero and the model reduces to the Daher-Mirman (henceforth DM, 2004) model which studies only the competition in the stock sector. Note that in the "centered" case, the equilibrium always exists (the second order condition is satisfied) and is symmetric (the trading levels of the two insiders exchanged in the stock market are the same). Moreover, the effect of the compensation received by the manager, as in JMC, is to center the profits of the manager around zero. Since the owner has no managerial tasks, he does not receive compensation and, thus, his profit function, as in Kyle (1985), is always centered around $\bar{z}$, the mean of $\tilde{z}$.

Remark 3.3. First, note that $B$ is positive. Second, the intercept coefficient of the price function, $\mu_{0}$, is different from zero. In contrast to JMC, this result is similar to DM. This result is due to the presence of competition in the stock sector.

The unique linear equilibrium of this model is presented and characterized in the next Proposition.

Proposition 2. A linear equilibrium exists. The equilibrium is unique and is characterized by,

(i) $y_{1}=\frac{\left(a+2 \mu_{1} b\right)}{3 b}$ and $y_{2}=\frac{\left(a-\mu_{1} b\right)}{3 b}$,

(ii) $x_{1}(\tilde{z})=\frac{(a-b Y)\left(y_{1}-\mu_{1}\right) \tilde{z}}{3 \mu_{2}}$ and $x_{2}(\tilde{z})=\frac{(a-b Y)\left(y_{1}-\mu_{1}\right) \tilde{z}-3 B}{3 \mu_{2}}$,

(iii) $p(\tilde{q}, \tilde{r})=\mu_{0}+\mu_{1} \tilde{q}+\mu_{2} \tilde{r}$,

(iv) $\mu_{0}=B=\frac{(a-b Y)\left(y_{1}-\mu_{1}\right)}{3} \bar{z}$,

(v) $\mu_{1}=\frac{a}{b} k$ and $\mu_{2}=\frac{\sqrt{2} a^{2} \sigma_{\varepsilon} \sqrt{(1-k)^{3} k}}{9 b \sigma_{u}}$,

where $k=\frac{\sigma_{z}^{2}}{\left(\sigma_{z}^{2}+9 \sigma_{\varepsilon}^{2}\right)}, \mu_{1} \in\left(0, \frac{a}{b}\right)$ and $\mu_{2}>0$.

Note that adding another informed trader to JMC changes the equilibrium outcome. In particular, in contrast to JMC, the compensation scheme of the manager must be equal to $\mu_{0}$. Moreover, there is an important informational affect, due to the presence of competition in the financial and the real sectors, on the equilibrium outcome of the two signals observed by the market maker. Indeed, Proposition 2 shows that the real signal 
and the total order flow signal, are respectively,

$$
\tilde{q}=\alpha(\tilde{z}+\tilde{\varepsilon})
$$

and

$$
\tilde{r}=\beta+\gamma \tilde{z}+\tilde{u},
$$

where $\alpha=(a-b Y), \beta=\frac{-B}{\mu_{2}}$ and $\gamma=\frac{2(a-b Y)\left(y_{1}-\mu_{1}\right)}{3 \mu_{2}}$. Here, $\alpha$ and $\gamma$ are the deterministic parts of $\tilde{q}$ and $\tilde{r}$, respectively, while $(\tilde{z}+\tilde{\varepsilon})$ and $\tilde{z}$ are the random parts.

Note that the noise in the total order flow signal, $\tilde{u}$ (equation 17), is additive and homoskedastic. In particular, an increase in the number of insiders, leads to an increase of the deterministic part of the total order flow signal, $\gamma$, i.e., the slope of the total order flow signal increases. In other words, increasing the number of insiders increases the slope of the insider trading in this paper compared to JMC. Moreover, this is done without influencing the variance of the noise term $\tilde{u}$. Hence, $\tilde{r}$ becomes more informative relative to JMC. In contrast, the deterministic part of the real signal $\alpha$ multiplies the sum of the random part, $(\tilde{z}+\tilde{\varepsilon})$ (equation 16). Therefore, the noise term in the real signal, $\tilde{\varepsilon}$ is heteroskedastic (see Creane 1994) but in such a way that changing the deterministic part of the real signal has no informational effect. In other words, changing the real signal coefficient $(a-b Y)$, changes the variance of $\tilde{q}$. Thus, the information change resulting from the change in $(a-b Y)$ is offset by the change of the variance of the real signal. Consequently, as in Tighe (1989), adding another informed trader, increases the information of the market maker but adding a competitive firm does not change the information incorporated in the stock price.

\section{Comparative Statics Analysis}

In this section we compare the equilibrium outcome presented in Proposition 2 to JMC and DM respectively. Note that, throughout this paper, "jmc" refers to JMC and "dm" to DM.

4.1. Equilibrium Outcome. In this section, we analyze the effect of both competition in the real and financial sectors on the equilibrium outcome. First, we compare the equilibrium outcome of our model, i.e in the presence of competition on both markets, to the equilibrium outcome in JMC, i.e. when only the competition in the real sector is taken into account. Second, we compare the equilibrium outcome of our model to the equilibrium outcome in DM, i.e., in the presence of competition in the financial sector.

Lemma 2. In the presence of competitions in both the real and financial markets, compared to $J M C$, we have,

(i) $\mu_{0}^{j m c}=0<\mu_{0}, \quad \mu_{1}<\mu_{1}^{j m c}$,

(ii) $\begin{cases}\mu_{2}>\mu_{2}^{j m c}, & \text { when } \sigma_{\varepsilon}^{2} \text { is small relative to } \sigma_{z}^{2}, \\ \mu_{2}<\mu_{2}^{j m c}, & \text { otherwise, }\end{cases}$

(iii) $y_{1}<y_{1}^{j m c}, y_{2}>y_{2}^{j m c}, Y<Y^{j m c}$, 
(iv) $x_{1}(z)<x^{j m c}(z)$

(v) $B\left(\mu_{1}\right)<B^{j m c}\left(\mu_{1}\right)$.

Proof: Recall that $\mu_{0}^{j m c}=0$, which is less than $\mu_{0}$ (by Lemma 1), and

$$
\mu_{1}^{j m c}=\frac{a \sigma_{z}^{2}}{b\left(\sigma_{z}^{2}+6 \sigma_{\varepsilon}^{2}\right)} .
$$

Comparing $\mu_{1}^{j m c}$ to $\mu_{1}$, the result in $(i)$ is proved. It is easy to prove $(i i)$ (use $(i)$ ) and it is straightforward to prove (iii) by using $(i)$ and (ii). Substituting the value of $\mu_{0}, \mu_{1}, \mu_{2}, y_{1}$ and $y_{2}$ in the expressions of $x_{1}($.$) and x_{2}($.$) from Proposition 2, we find that,$

$$
x_{1}(z)=\frac{\sqrt{2} \sigma_{u}}{2 \sigma_{z}} z
$$

which is less than the level of trading of the manager in JMC, that is,

$$
x^{j m c}(z)=\frac{\sigma_{u}}{\sigma_{z}} z .
$$

Hence, $(i v)$ is proved. It is easy to check that,

$$
B\left(\mu_{1}\right)=\frac{\left(a-b \mu_{1}\right)^{2}}{27 b} \bar{z} \text { and } B^{j m c}\left(\mu_{1}\right)=\frac{\left(a-b \mu_{1}\right)^{2}}{18 b} \bar{z},
$$

which proves $(v)$.

Lemma 3. Comparing the owner's level of trading, as well as the total level of trading of the two insiders, to the level of trading in $J M C$, we have,

(i) there exists a $z^{-} \in \mathbb{R}^{-}$such that,

$$
\begin{cases}x_{2}(z)>x^{j m c}(z), & \text { if } z \in\left(z^{-}, 0\right) \\ x_{2}(z)<x^{j m c}(z), & \text { otherwise, }\end{cases}
$$

(ii) there exists $a z^{+} \in \mathbb{R}^{+}$such that,

$$
\begin{cases}x(z)<x^{j m c}(z), & \text { if } z \in\left(0, z^{+}\right) \\ x(z)>x^{j m c}(\tilde{z}), & \text { otherwise. }\end{cases}
$$

Proof: Substituting for $Y, y_{1}, \mu_{1}$ and $\mu_{2}$ from Proposition 2 into the expression for $x_{2}($. yields,

$$
x_{2}(z)=\frac{\sqrt{2} \sigma_{u}}{2 \sigma_{z}}(z-\bar{z}) .
$$

Comparing the expressions in (18) and (19), the result in $(i)$ holds. By adding (18) and (19), we obtain,

$$
x(z)=\frac{\sqrt{2} \sigma_{u}}{\sigma_{z}} z-\frac{\sqrt{2} \sigma_{u}}{2 \sigma_{z}} \bar{z} .
$$

Comparing equation (18) to equation (20), we get the result. 
Before interpreting these results, the relationship between the basic Jain-Mirman model (henceforth JM, 2000), JMC, DM and the model of this paper should be pointed out. Our model adds a second informed trader to the model of JMC, so there is competition in both the real and the financial sectors. On the other hand, DM adds a second inside trader to JM so that there is competition in the financial sector but not in the real sector. In both our model and in DM the second inside trader has no managerial responsibilities but has the same information as the manager. Hence, not only can we study the effect of competition among insiders on the variables of the model, but we can also study the effect that competition in the real sector has on competition in the stock sector and visa versa.

To begin, note that the stock price set by the market maker, is affected by both competition in the real sector and competition in the financial sector. Lemma $2(i)$, shows that the intercept coefficient of the stock price, $\mu_{0}$, is greater than in JMC. Indeed, to satisfy the second order condition of the maximization problem of the manager of firm 1 , and to guarantee the existence of an equilibrium as in DM, competition in the financial sector requires that the compensation scheme received by the manager (which is positive) has the same value as $\mu_{0}$. In the absence of this competition in the financial sector, i.e., in $\mathrm{JM}$ or JMC, $\mu_{0}$ must be equal to zero. Hence, $\mu_{0}$ is set by the market maker in order to satisfy the second order condition of the manager. Then, given the value of $\mu_{0}$, the market maker sets the value of $\mu_{1}$ and $\mu_{2}$ to satisfy the zero profits condition.

Second note that $\mu_{1}$, the response of the market maker to the real signal, is always less than in JMC, while the response of the market maker to the total order flow signal $\mu_{2}$ depends on the variances of the exogenous variables. In other words, $\mu_{2}$ is lower in this model than in JMC when the variance of the noise from the real sector, i.e. $\sigma_{\varepsilon}^{2}$, is large relative to the variance of the value of the firm $\sigma_{z}^{2}$. This relationship, between $\mu_{1}$ and $\mu_{2}$, is similar to DM (compared to JM) as well as in the Jain-Mirman (henceforth JMJ, 1999) model going from one to two insiders, each with the same information. Indeed it is this result of JMJ that gives the basis for understanding the effect of adding another insider. JMJ is a Kyle-type model (with one insider) in which the market maker observes two signals, i.e., the total order flow and $\tilde{z}+\tilde{\varepsilon}$, where $\tilde{z}$ is the liquidation value of the asset and $\tilde{\varepsilon}$ is a noise term. Since the market maker observes the two signals, the price is set in the same way as in JM but with no real market so that the value of the asset is the information of each insider. When competition between two insiders is studied in JMJ, the relationship between $\mu_{1}$ and $\mu_{2}$, is similar to the result in our model. In other words, going from one to two insiders, $\mu_{1}$ decreases while $\mu_{2}$ depends on the variances of the exogenous variables. This result reflects the fact that with two insiders there is more information in the order flow signal and, thus, the order flow signal gets, in general, more weight, except when there is so much noise in the real signal that it becomes like the one signal Kyle model. The result is then similar to the Kyle model, i.e., $\mu_{2}$ decreases. However, with production, although the results are similar to the JMJ results, the value of 
the firm has two components, the value of the random variable $\mathrm{z}$ (which is the information in Kyle) and the deterministic real part of the firm.

In fact, $\mu_{2}$ is lower in this model than in JMC, when $\sigma_{\varepsilon}^{2}$, is large relative to $\sigma_{z}^{2}$. This result is similar to the result in Kyle's model going from one to two insiders (see in Tighe 1989) and is consistent with the results of JMJ. In order to understand how this coefficient varies, recall that

$$
\mu_{2}=\frac{\sigma_{v r} \sigma_{q}^{2}-\sigma_{v q} \sigma_{q r}}{\sigma_{q}^{2} \sigma_{r}^{2}-\left(\sigma_{q r}\right)^{2}}
$$

Hence the value of $\mu_{2}$ in this paper and in JMC are, respectively,

$$
\mu_{2}=\frac{3 \sqrt{2} a^{2} \sigma_{\varepsilon}^{4} \sigma_{z}}{b \sigma_{u}\left(\sigma_{z}^{2}+9 \sigma_{\varepsilon}^{2}\right)^{2}} \text { and } \mu_{2}^{j m c}=\frac{2 a^{2} \sigma_{\varepsilon}^{4} \sigma_{z}}{b \sigma_{u}\left(\sigma_{z}^{2}+6 \sigma_{\varepsilon}^{2}\right)^{2}} .
$$

Increasing $\sigma_{\varepsilon}^{2}$ relative to $\sigma_{z}^{2}$ increases the variance of the real signal, $\sigma_{q}^{2}$, as well as the covariance of the real signal and the total order flow, $\sigma_{q r}$. Hence, in this case, the numerator increases less than the denominator, thus yielding a lower value for $\mu_{2}$ in this model relative to JMC.

Moreover the relationship between $\mu_{2}$ and the variances of the exogenous variables can also be inferred from the zero expected profit condition. Indeed, recall the expression of the stock price in each model. We have in JMC, at equilibrium,

$$
p(\tilde{q}, \tilde{r})=E[\tilde{v} \mid \tilde{q}, \tilde{r}]=\mu_{1}^{j m c} \tilde{q}+\mu_{2}^{j m c} \tilde{r} .
$$

So taking expectation on both sides of the equality we obtain,

$$
\bar{v}^{j m c}=\mu_{1}^{j m c} \bar{q}+\mu_{2}^{j m c} \bar{r},
$$

which is equivalent to

$$
(a-b Y) y_{1} \bar{z}-B^{j m c}=\mu_{1}^{j m c} \bar{q}+\mu_{2}^{j m c} \bar{r}
$$

or

$$
(a-b Y) y_{1} \bar{z}=B^{j m c}+\mu_{1}^{j m c} \bar{q}+\mu_{2}^{j m c} \bar{r} .
$$

A similar calculation for this paper, taking account of the fact that $\mu_{0}$ is different from zero and is equal to the compensation scheme yields,

$$
(a-b Y) y_{1} \bar{z}=2 B+\mu_{1} \bar{q}+\mu_{2} \bar{r} .
$$

When $\sigma_{\varepsilon}^{2}$ is large relative to $\sigma_{z}^{2}$, the left hand sides of equations (21) and (22) are very close in value and then the right hand sides must be close. In this case $\mu_{2}$ is less than in JMC, since the unconditional profits of the two firms are close and the real signal is very noisy. This is similar to the Kyle result that the coefficient of the order flow decreases since insider trading increases and the value of the firms are the same.

In contrast, when $\sigma_{\varepsilon}^{2}$ is small relative to $\sigma_{z}^{2}$, the total order flow signal coefficient, $\mu_{2}$ in this model is greater than JMC. The intuition for this result is that the same consideration as in JMJ happens here i.e., the demand for the stock together with the zero expected 
profit condition for the market maker, reduce $\mu_{1}$ but in this case, $\mu_{2}$ increases since it is now more informative. Moreover, the unconditional profits of the firm 1 in JMC are less than the unconditional profits of firm 1 in our paper. Since the real signal coefficient is reduced, the more informative order flow coefficient increases to reflect the increased value of the firm. In order to understand this relationship between the stock price coefficients and the unconditional profits of the firm 1, consider the expression of the unconditional profits of firm 1 in JMC and in our model presented in equations (21) and (22), respectively. These two equations have the following expression,

$$
(a-b Y) y_{1} \bar{z}-B^{j m c}=\mu_{1}^{j m c} \bar{q}+\mu_{2}^{j m c} \bar{r}
$$

and

$$
(a-b Y) y_{1} \bar{z}-B=\mu_{0}+\mu_{1} \bar{q}+\mu_{2} \bar{r} .
$$

Hence, when $\sigma_{\varepsilon}^{2}$ is small relative to $\sigma_{z}^{2}$, the left hand side of (23) is less than the left hand side of (24) and then the right hand side of (23) must be less than the right hand side of (24). This increase in profits, given that $\mu_{1}$ decreases relative to JMC can be absorbed by giving the more informative signal more weight thus making $\mu_{2}$, greater than in JMC.

Third, competition in the financial sector has an effect on the output of firm 1 . In fact, the level of output produced by firm 1 is less than in JMC. To see this, recall the expression of the output produced by firm 1 ,

$$
y_{1}=\frac{a+\mu_{1} b}{3 b} \text {. }
$$

Hence, the output is a function of $\mu_{1}$, the coefficient of the real sector signal of the stock price, which is set by the market maker. Lemma $2(i)$ shows that competition in the stock sector, reduces the value of $\mu_{1}$ and thus the output of firm 1 in our model relative to JMC. Moreover, due to the movement along the reaction curve of firm 2, the level of output of firm 2 is greater than in JMC. Consequently, total output in this model is less than in JMC. Hence, the addition of another informed trader influences the production decision of the manager of firm 1 , as well as the production decision of firm 2 .

Fourth, competition in the stock sector affects the insiders trades. Indeed, Lemma 2 (iv) shows that the manager's trading level is less than in JMC. This result is also consistent with Kyle (1985), going from one to two insiders (see in Tighe 1989). Moreover, from equation (18), the manager's trading level, as in JMC, is a linear function on $\tilde{z}$. This is due to the presence of the compensation scheme that makes the form of the trading function of the manager linear through the origin. ${ }^{6}$ Moreover, competition in the financial sector also affects the trading level of the owner, as well as the total trade. Both of these trades are affine functions on $\tilde{z}$, due to the influence of the compensation scheme. Since the owner has no managerial responsibilities in the real sector, he does not receive compensation.

\footnotetext{
${ }^{6}$ Note that the compensation scheme received by the market maker pushes the trading level function to be linear through the origin and thus his profits are independent from the prior beliefs of the market maker.
} 
Thus, his trading level has the same form as the trading level of the insider in a Kyle-type model (see Tighe 1989).

Finally, the comparison between the compensation schemes in both models depends on the variances of the exogenous variables. Specifically, higher values of the variance of the noise signal of the real sector, $\sigma_{\varepsilon}^{2}$, relative to $\sigma_{z}^{2}$, makes the compensation in our model less than in JMC. In contrast, when $\sigma_{\varepsilon}^{2}$ is small, the compensation in our model is greater than in JMC. The interpretation for this result is that when $\sigma_{\varepsilon}^{2}$ is small, relative to $\sigma_{z}^{2}$, the unconditional net profits of firm 1 in JMC are less than in our paper and, hence, the manager in JMC is less compensated than in our model. In contrast, when $\sigma_{\varepsilon}^{2}$ is large, relative to $\sigma_{z}^{2}$, the total order flow signal becomes more informative relative to the real signal, since its variance is also large. This case is consistent with Kyle-type models, going from one to two insiders. ${ }^{7}$ It should be pointed out that the comparison of the compensation schemes, as $\sigma_{\varepsilon}^{2}$ varies relative to $\sigma_{z}^{2}$ between JMC and this paper is similar to the comparison between the coefficient of the total order flow signal, $\mu_{2}$ as $\sigma_{\varepsilon}^{2}$ varies relative to $\sigma_{z}^{2}$, between JMC and this paper (see Lemma 2) .

Now, we compare the equilibrium outcome of this model to the equilibrium outcome in DM, i.e., when only competition in the financial sector is studied.

Lemma 4. Comparing to DM,

(i) $\mu_{0}<\mu_{0}^{d m}, \quad \mu_{1}<\mu_{1}^{d m}, \quad \mu_{2}<\mu_{2}^{d m}$,

(ii) $y_{1}<y^{d m}, \quad Y>y^{d m}$

(iii) $x_{1}(\tilde{z})=x_{1}^{d m}(\tilde{z}), \quad x_{2}(\tilde{z})=x_{2}^{d m}(\tilde{z})$,

(iv) $B<A^{d m}$

Proof: Recall that $\mu_{1}^{d m}$ has the same expression as $\mu_{1}^{j m c}$. Hence, by Lemma 2, the inequality in the middle of $(i)$ is proved. Since, $\mu_{0}$ takes the same value as the compensation scheme, we prove $(i v)$, which is equivalent to the left hand side of $(i)$. Indeed, it is easy to check that the values of $B$ and $A$ are respectively,

$$
B=\frac{3 a^{2} \sigma_{\varepsilon}^{4}}{b\left(\sigma_{z}^{2}+9 \sigma_{\varepsilon}^{2}\right)^{2}} \bar{z} \text { and } A^{d m}=\frac{3 a^{2} \sigma_{\varepsilon}^{4}}{b\left(\sigma_{z}^{2}+6 \sigma_{\varepsilon}^{2}\right)^{2}} \bar{z} .
$$

Comparing these two values, $(i v)$ is then proved. It is straightforward to prove the right hand side of $(i)$ (use the middle and the left hand side of $(i)$ ). Comparing the level of output produced by firm 1 and the total outputs in this model to DM, we obtain $(i i)$. Finally, recall that the values of the insiders' trading orders of DM are the same as in equations (18) and (19). Thus, (iii) is proved.

\footnotetext{
${ }^{7}$ When the market maker receives only one signal, i.e., the total order flow signal, as in Kyle, then, the greater is the number of "owners", each with same information, the smaller is the compensation scheme received by the manager.
} 
Lemma 4 shows how competition in the real sector affects the equilibrium outcome when competition in the stock sector is taken into account.

It should be noted that competition in the real sector adds no new information, ${ }^{8}$ but does lower the profits of the firm due to Cournot competition in the real sector. These lowered profits must be incorporated in the stock price function of the market maker. Also, the compensation scheme is affected by competition in the financial sector. In particular, without competition in the financial sector and adding a second firm in the real sector has no effect on the value of $\mu_{0}$, since $\mu_{0}=0$, in both JM and JMC. But the addition of a second insider changes the value of $\mu_{0}$ (to satisfy the second order condition of the manager) by making it equal to the compensation scheme. Thus, the effect of adding competition in the real sector depends on the financial aspects of the firm, in particular, it depends on the number of insiders trading on the stock market. However, the major effect of a competition in the real sector is to decrease profits without increasing the amount of information.

Note that Lemma 4 shows that the coefficients of the stock price function, in our model, are all less than DM. In order to understand this result, recall the expression of the "net" unconditional expected value of the firm 1 in each model. For DM,

$$
\bar{v}^{d m}=\left(a-b y^{d m}\right) y^{d m} \bar{z}-A^{d m}=\mu_{0}+\mu_{1}^{d m} \bar{q}+\mu_{2}^{d m} \bar{r} .
$$

The unconditional expected value of firm 1 in our model is,

$$
\bar{v}=(a-b Y) y_{1} \bar{z}-B=\mu_{0}+\mu_{1} \bar{q}+\mu_{2} \bar{r} .
$$

The left hand sides in equations (26) and (25) are, respectively,

$$
\frac{3 a^{2} \sigma_{\varepsilon}^{2}\left(\sigma_{z}^{2}+2 \sigma_{\varepsilon}^{2}\right)}{b\left(\sigma_{z}^{2}+6 \sigma_{\varepsilon}^{2}\right)^{2}} \text { and } \frac{3 a^{2} \sigma_{\varepsilon}^{2}\left(\sigma_{z}^{2}+2 \sigma_{\varepsilon}^{2}\right)}{b\left(\sigma_{z}^{2}+9 \sigma_{\varepsilon}^{2}\right)^{2}} .
$$

This means that the unconditional net profit of firm 1 in this model is less than in DM. Hence, the right hand side of (25) must be less than the right hand side of (26). In this paper, the manager is compensated less than in DM, and hence the value of $\mu_{0}$ in this paper is less than in DM. Together with the fact that $\bar{r}$ is the same in both models (by Lemma $4($ iii $)$ ) and the fact that $\bar{q}$ in this paper is less than in DM, we obtain that $\mu_{1}$ and $\mu_{2}$ are both less than in DM. In sum, competition on the real sector reduces the unconditional expected value of firm 1 and, thus, the coefficients of the stock price signals are less with the presence of the real sector competition than in the absence of this competition. Note that this result is similar to the result comparing $\mathrm{JMC}$ to $\mathrm{JM}^{9}$

\footnotetext{
${ }^{8}$ It is shown in the paragraph after Proposition 2, that the noise term in the real signal is heteroskedastic, and that the informational content of the stock price does not change in this model, relative to DM

${ }^{9}$ Note that in JMC the value of $\mu_{2}$ is less than in JM. The difference between the result stated in this paper and the result reported in JMC comes from the calculation of $\mu_{1}$. After correcting the value of $\mu_{1}$ in $\mathrm{JMC}$, the value of $\mu_{2}$ in $\mathrm{JMC}$ is less than in JM
} 
Second, the output produced by firm 1 is less than in DM and the total output is greater than in DM. This is true for two reasons. First, since $\mu_{1}$ has an effect on the output, the reduction in $\mu_{1}$ lowers the output of firm 1. Second due to the Cournot duopoly framework, the output of firm 1 also decreases due to the existence of the second firm. Thus the two effects together reduce the output of firm 1. The total output of the two firms increases due to Cournot competition, and, thus the price and the gross profit (revenue) of firm 1 decreases.

Third, note that the trading function of each insider is the same as in DM, although the financial asset value, i.e., the profits of the firm, is different in both models. In part, this is due to the fact that the market maker takes into account the changes in the market structure (since the output produced by firm 1 as well as the total outputs are deterministic). This is also due to the zero expected profit condition of the market maker (which requires that the price is equal to the conditional expectation of the asset's value given the signals he observes). In other words, at equilibrium each insider trades the same quantity as in DM in order to maximize his profits.

4.2. Information Revelation. We start this section by evaluating the information revelation in this model relative to JMC and DM. Indeed, we show that adding another insider increases the informational content of the stock price compared to JMC. In contrast, adding a competitive firm in the real sector, the level of the information revealed by the stock price set by the market maker remains unchanged relative to DM. Hence, the information incorporated in the stock price is affected by the presence of competition in the financial sector and not competition in the real sector. In order to interpret these results we use, as measure of informativeness, the conditional variance of $\tilde{z}$ given the information of the market maker. ${ }^{10}$ This can be written as, ${ }^{11}$

$$
\operatorname{Var}(\tilde{z} \mid \tilde{q}, \tilde{r})=\frac{\left(y_{1}-\mu_{1}\right)}{3 y_{1}} \sigma_{z}^{2},
$$

substituting the expressions of $y_{1}$ and $\mu_{1}$ of Proposition 2, we get,

$$
\operatorname{Var}(\tilde{z} \mid \tilde{q}, \tilde{r})=\frac{\sigma_{\varepsilon}^{2}}{\sigma_{z}^{2}+3 \sigma_{\varepsilon}^{2}} \sigma_{z}^{2} .
$$

Proposition 3. The stock price reveals more information in this model than in JMC and the same amount of information as in DM.

Proof: Recall that the conditional variance in JMC and DM are, respectively,

$$
\frac{\sigma_{\varepsilon}^{2}}{\sigma_{z}^{2}+2 \sigma_{\varepsilon}^{2}} \sigma_{z}^{2} \text { and } \frac{\sigma_{\varepsilon}^{2}}{\sigma_{z}^{2}+3 \sigma_{\varepsilon}^{2}} \sigma_{z}^{2}
$$

\footnotetext{
${ }^{10}$ Since there is no real market in Kyle (1985), the value of the financial asset is the information of the insider. In contrast, in JM, JMC, DM and in this paper, the information of the insider is not the value of the firm.

${ }^{11}$ See Theorem 1 in the Appendix
} 
Comparing to equation (27), the result is proved .

The intuition for this result is the following. First, the noise term $\varepsilon$, in the real signal is heteroskedastic and multiplicative (see equation 16) and therefore, the information of the market maker is the same when the deterministic part $\alpha$ of the real signal increases. Hence, adding a second firm to DM, increases the deterministic part of the real signal without changing the deterministic part $\gamma$ of the total order flow signal observed by the market maker (Lemma 4) and thus the information transmitted to the market maker through both signals remains unchanged.

Second, note that the noise term $\tilde{u}$, in the total order flow signal is homoskedastic (see equation 17). Hence, the greater the deterministic part $\gamma$ of the total order flow signal, the more informative is the total order flow signal. Adding a second informed trader to JMC, i.e, the model of our paper, changes the values of the deterministic part $\alpha$, of the real signal as well as the deterministic part $\gamma$, of the total order flow signal observed by the market maker. Hence, when the market maker observes these two signals, he receives more information about $z$ only through the total order flow since the the noise of the real is heteroskedastic and multiplicative. Consequently, adding a competition in the financial sector increases the level of information incorporated in the stock price set by the market maker. Note that this result is consistent with Kyle-type model going from one to two insiders (see Tighe 1989).

\section{Insiders Profits}

In this section, we analyze the profits of the two insiders. First, we show that the profits of the manager are not always less than the profits of the manager in JMC. Second, relative to DM, we prove that the profits of the manager and the owner of this model are less than in DM.

5.1. Manager's Profits. We show, in this section, that the manager's conditional profits in our model are always less than in DM. On the other hand, the conditional profits of the manager in JMC are less than our model when $\sigma_{\varepsilon}^{2}$ is small, relative to $\sigma_{z}^{2}$ and greater than in JMC, otherwise. To interpret these results, we calculate the profits of the manager in this model. Substituting for $Y, x_{1}(),. x_{2}(),. \mu_{0}, \mu_{1}, \mu_{2}$ from Proposition 2 , in the manager's conditional profit function, we obtain,

$$
G_{1}(\tilde{z})=\frac{3 \sqrt{2} a^{2}\left(\sigma_{\varepsilon}^{2}\right)^{2} \tilde{z}^{2} \sigma_{u}}{2 b \sigma_{z}\left(\sigma_{z}^{2}+9 \sigma_{\varepsilon}^{2}\right)^{2}} .
$$

Recall that the insider's profits in JMC and DM are respectively,

$$
G_{1}^{j m c}(\tilde{z})=\frac{2 a^{2}\left(\sigma_{\varepsilon}^{2}\right)^{2} \tilde{z}^{2} \sigma_{u}}{b \sigma_{z}\left(\sigma_{z}^{2}+6 \sigma_{\varepsilon}^{2}\right)^{2}} .
$$


and

$$
G_{1}^{d m}(\tilde{z})=\frac{3 \sqrt{2} a^{2}\left(\sigma_{\varepsilon}^{2}\right)^{2} \tilde{z}^{2} \sigma_{u}}{2 b \sigma_{z}\left(\sigma_{z}^{2}+6 \sigma_{\varepsilon}^{2}\right)^{2}}
$$

The following Proposition, shows that the manager's conditional profits in our model are less than in DM.

Proposition 4. $G_{1}<G_{1}^{d m}$

The essence of this result is that, relative to DM, competition in the real sector reduces the net unconditional profits $\bar{v}$, which is the value of the financial asset of firm 1 . To understand the relationship between the value of the firm and the profits of the manager, recall that the expressions of the manager's conditional profits in our model and DM are, respectively,

$$
G_{1}(\tilde{z})=\frac{\left[(a-b Y)\left(y_{1}-\mu_{1}\right) \tilde{z}\right] x_{1}(\tilde{z})}{3} \text { and } G_{1}^{d m}(\tilde{z})=\frac{\left[\left(a-b y^{d m}\right)\left(y^{d m}-\mu_{1}^{d m}\right) \tilde{z}\right] x_{1}^{d m}(\tilde{z})}{3}
$$

Indeed, the expression of the manager's conditional profits is a product of the trading level of the manager times a function, i.e., $g(\tilde{z})=\frac{\left[(a-b Y)\left(y_{1}-\mu_{1}\right) \tilde{z}\right]}{3}$ of $\tilde{z}$. Since the trading level of the manager is the same in both models (see Lemma 4), the same lemma shows that $g(\tilde{z})$ in our model is less than in DM. Consequently, the Cournot duopoly structure reduces the manager's profits in this model relative to DM.

Now we show, in the next Proposition, that relative to JMC, the manager's profits are not always less his profits in JMC. We have,

Proposition 5. $\begin{cases}G_{1}>G_{1}^{j m c} & \text { when } \sigma_{\varepsilon}^{2} \text { is small relative to } \sigma_{z}^{2}, \\ G_{1}<G_{1}^{j m c} & \text { otherwise }\end{cases}$

Proof: The proof is obvious and thus omitted

The intuition for this result is that for small values of $\sigma_{\varepsilon}^{2}$, relative to $\sigma_{z}^{2}$, the unconditional net profits of firm 1 (equations 21 and 22), in JMC are less than in our model. Thus, the conditional profits of the manager in JMC are less than in our model. In contrast, when $\sigma_{\varepsilon}^{2}$ is large, relative to $\sigma_{z}^{2}$, the net unconditional profits of firm 1 in our paper become less than in JMC and so the conditional profits of the manager in JMC are greater than in our model. ${ }^{12}$

\footnotetext{
${ }^{12}$ The relationship between JM and JMC, going from one to two firms producing in the real sector yields the intuitive result that profits are reduced, due to Cournot competition. However, going from one to two insiders, i.e., JM to DM has an ambiguous effect on profits, in some cases from increasing the variance of the exogenous variables and from decreasing them in other cases. However, it would appear that going from one insider and one real firm to two insiders and two real firms, i.e., JM to this paper, that profits should also decline due to Cournot competition, i.e., as going from JM to JMC. Moreover this is not the case. In fact, the effect on profit due to the addition of a second firm depends upon the number of insider trading. Indeed, in this situation profits sometimes decline and sometimes increase, as it does going from JM to DM.
} 
It should be pointed out that adding a second firm, which does not change the information content of the two signals (Proposition 3), reduces the profits of the firm. This is due, mostly to Cournot competition. However adding another informed trader increases the informational content of the total order flow signal but has a minor effect, compared to adding a second firm, on the profitability of the firm. Note that adding a second insider to JMC, Proposition 5 is relative to the result of the effect of $\mu_{2}$ in Lemma 2 as well as the compensation scheme received by the manager.

Remark 5.1. If the market maker receives only one signal (the total order flow), the conditional profits of the manager in our model are less than JMC, which are less than DM. This result is due to the fact that the unconditional profits of the firm in this paper are less than in JMC as well as in DM, when only the total order is observed. ${ }^{13}$

Remark 5.2. Note that the compensation scheme received by the manager makes his profits to be independent of the prior beliefs of the market maker, i.e, $\bar{z}$. On the other hand, the profits of the owner depend on the prior beliefs of the market maker, a result similar to JMJ, going from one to two insiders, and to Tighe. This is due to the fact that the owner has no managerial activities and, thus, he does not receive a compensation scheme. ${ }^{14}$

5.2. Owner's Profit. In this section, we compare the owner's conditional profits in this model to the owner's conditional profits in DM. We show that under competition in both the real and the financial markets, the conditional profits of the owner are less than the owner's profits in DM.

In order to interpret these results, we calculate the profits of the manager in this model. Substituting for $Y, x_{1}(),. x_{2}(),. \mu_{0}, \mu_{1}, \mu_{2}$ from Proposition 2 , in the manager's profits function, we obtain,

$$
G_{2}(\tilde{z})=\frac{3 \sqrt{2} a^{2}\left(\sigma_{\varepsilon}^{2}\right)^{2}(\tilde{z}-\bar{z})^{2} \sigma_{u}}{2 b \sigma_{z}\left(\sigma_{z}^{2}+9 \sigma_{\varepsilon}^{2}\right)^{2}}
$$

Recall that the owner's profits in DM is,

$$
G_{2}^{d m}(\tilde{z})=\frac{3 \sqrt{2} a^{2}\left(\sigma_{\varepsilon}^{2}\right)^{2}(\tilde{z}-\bar{z})^{2} \sigma_{u}}{2 b \sigma_{z}\left(\sigma_{z}^{2}+6 \sigma_{\varepsilon}^{2}\right)^{2}} .
$$

\footnotetext{
${ }^{13}$ Indeed, it is shown in DM that the conditional expected profits of the manager in JMC are less than in DM when the market maker observes only the total order flow. Moreover, it is easy to check that in this case, i.e., when the market maker observes only the total order flow, the profits of the manager in this model are also less than in JMC.

${ }^{14}$ It should be pointed out that in JMJ going from one two insiders, the market maker also observes two signals. But, the absence of the real market in JMJ yields a symmetric equilibrium and thus an equal profits each dependent on the prior beliefs of the market maker. This is not the case in this paper, since the real market is taken into account and, thus, a compensation schemes is required to satisfy the second order condition of the manager.
} 
A comparison between the expressions of the owner's profits in this model and in DM, leads to the following result.

Proposition 6. Conditional profits of the owner in our model are less than in DM, that is, $G_{2}<G_{2}^{d m}$

The intuition for this result, is that adding a competitive firm in the real sector reduces the net value of the firm 1 and then reduces the conditional profits of the owner relative to DM.

Remark 5.3. If the market maker receives only the total order flow as in Kyle, the conditional profits of the owner in this paper continue to be less than in DM. This is due to Cournot competition which lowers the profits of firm 1.

\section{APPENDIX}

We start this appendix by recalling the Theorem that we use to prove Lemma 1 . Then we prove this Lemma.

Theorem 1. If the $p \times 1$ vector $Y$ is normally distributed with mean $U$ and covariance $V$ and if the vector $Y$ is partitioned into two subvectors such that $Y=\left(\begin{array}{c}Y_{1} \\ Y_{2}\end{array}\right)$ and if $Y^{*}=\left(\begin{array}{c}Y_{1} \\ Y_{2}^{*}\end{array}\right)$

$$
U=\left(\begin{array}{c}
U_{1} \\
U_{2}
\end{array}\right) \text { and } V=\left(\begin{array}{ll}
V_{11} & V_{12} \\
V_{21} & V_{22}
\end{array}\right)
$$

are the corresponding partitions of $Y^{*}, U$ and $V$, then the conditional distribution of the $m \times 1(m<p)$ vector $Y_{1}$ given the vector $Y_{2}=Y_{2}^{*}$ is the multivariate normal distribution with mean $U_{1}+V_{12} V_{22}^{-1}\left(Y_{2}^{*}-U_{2}\right)$ and covariance matrix $V_{11}-V_{12} V_{22}^{-1} V_{21}$.

Proof: See Graybill, Theorem 3.10 pp 63.

\section{Proof of Lemma 1:}

We apply Theorem 1 to the normal random vector $B=(\tilde{v}, \tilde{q}, \tilde{r})$. First, in this case we have $p=3$ and $m=1$. Second by identification, we have $Y_{1}=\tilde{v}$ and $Y_{2}=\left(\begin{array}{c}\tilde{q} \\ \tilde{r}\end{array}\right) \cdot U_{1}=\bar{v}$, $U_{2}=\left(\begin{array}{c}\bar{q} \\ \bar{r}\end{array}\right)$ and

$$
V=\left(\begin{array}{ccc}
\sigma_{v}^{2} & \sigma_{v q} & \sigma_{v r} \\
\sigma_{v q} & \sigma_{q}^{2} & \sigma_{q r} \\
\sigma_{v r} & \sigma_{q r} & \sigma_{r}^{2}
\end{array}\right)=\left(\begin{array}{cc}
V_{11} & V_{12} \\
V_{21} & V_{22}
\end{array}\right)
$$

Where $V_{11}=\sigma_{v}^{2}, V_{12}=\left(\sigma_{v q}, \sigma_{v r}\right), V_{21}=\left(\begin{array}{c}\sigma_{v q} \\ \sigma_{v r}\end{array}\right)$ and $V_{22}=\left(\begin{array}{cc}\sigma_{q}^{2} & \sigma_{q r} \\ \sigma_{q r} & \sigma_{r}^{2}\end{array}\right)$. 
Note that

$$
V_{22}^{-1}=\frac{1}{D}\left(\begin{array}{cc}
\sigma_{r}^{2} & -\sigma_{q r} \\
-\sigma_{q r} & \sigma_{q}^{2}
\end{array}\right),
$$

where $D$ is the determinant of $V_{22}$, that is $D=\sigma_{q}^{2} \sigma_{r}^{2}-\sigma_{q r}^{2}$.

So we obtain,

$$
\begin{aligned}
& \mu_{0}=\bar{v}-\mu_{1} \bar{q}-\mu_{2} \bar{r} \\
& \mu_{1}=\frac{\sigma_{v q} \sigma_{r}^{2}-\sigma_{v r} \sigma_{q r}}{D} \\
& \mu_{2}=\frac{\sigma_{v r} \sigma_{q}^{2}-\sigma_{v q} \sigma_{q r}}{D}
\end{aligned}
$$

Substituting for the variances and covariances in (34) and (35), we get

$$
\begin{gathered}
\mu_{1}=\frac{(a-b Y)^{2} y_{1} \sigma_{z}^{2} \sigma_{u}^{2}}{D}, \\
\mu_{2}=\frac{2(a-b Y)^{4} y_{1}\left(y_{1}-\mu_{1}\right) \sigma_{z}^{2} \sigma_{\varepsilon}^{2}}{3 \mu_{2} D} .
\end{gathered}
$$

Computing (36) and (37), we obtain

$$
3 \mu_{2}^{2}=\frac{2(a-b Y)^{2} \mu_{1}\left(y_{1}-\mu_{1}\right) \sigma_{\varepsilon}^{2}}{\sigma_{u}^{2}}
$$

Calculating for the expression of $D$, we get

$$
D=\frac{4(a-b Y)^{4}\left(y_{1}-\mu_{1}\right)^{2} \sigma_{\varepsilon}^{2} \sigma_{z}^{2}}{9 \mu_{2}^{2}}+(a-b Y)^{2} \sigma_{u}^{2}\left(\sigma_{\varepsilon}^{2}+\sigma_{z}^{2}\right) .
$$

Substituting the above expression of $D$ in (37), we find

$$
3 \mu_{2}^{2}=\frac{2(a-b Y)^{2}\left(y_{1}-\mu_{1}\right)\left(y_{1}+2 \mu_{1}\right) \sigma_{\varepsilon}^{2} \sigma_{z}^{2}}{3 \sigma_{u}^{2}\left(\sigma_{\varepsilon}^{2}+\sigma_{z}^{2}\right)} .
$$

Solving (38) and (39), we get

$$
3 \mu_{1}\left(\sigma_{\varepsilon}^{2}+\sigma_{z}^{2}\right)=\sigma_{z}^{2}\left(y_{1}+2 \mu_{1}\right) .
$$

Substituting for $y$ to solve $\mu_{1}$, we obtain

$$
\mu_{1}=\frac{a \sigma_{z}^{2}}{b\left(\sigma_{z}^{2}+9 \sigma_{\varepsilon}^{2}\right)} .
$$

To solve for $\mu_{2}$, we substitute the above value of $\mu_{1}$ in (38) and taking the positive root, we get

$$
\mu_{2}=\frac{\sqrt{2} a^{2} \sigma_{\varepsilon} \sqrt{(1-k)^{3} k}}{9 b \sigma_{u}}
$$


where $k=\frac{\sigma_{z}^{2}}{\sigma_{z}^{2}+9 \sigma_{\varepsilon}^{2}}$.

\section{REFERENCES}

[1] A.Creane, Experimentation with Heteroskedastic Noise, Economic Theory, 4, (1994), 275-286.

[2] W. Daher and L.J. Mirman, Market Structure and Insider Trading, Working Paper, Cahier de la MSE, 2004.25, Université Paris I (2004).

[3] J. Dow and R.Rahi, Informed Trading, Investment and Welfare, Journal of Business, 76, (2003), 439-454.

[4] F.Graybill An Introduction to Linear Statistical Models: Volume I, McGraw Hill. 1961.

[5] C. Holden and A. Subrahmanyam, Long-Lived Private Information and Imperfect Competition, Journal of Finance. 47, (1992), 247-270.

[6] M.Manove, The Harm from Insider Trading and Informed Speculation, Quarterly Journal of Economics, 104,(1989), 823-845.

[7] N.Jain and L.J. Mirman, Real and Financial Effects of Insider Trading with Correlated Signals, Economic Theory. 16, (2000), 333-353.

[8] N.Jain and L.J. Mirman, Effects of Insider Trading under Different Market Stuctures, The Quarterly Review of Economics and Finance. 42, (2002), 19-39.

[9] N.Jain and L.J. Mirman, Insider Trading with Correlated Signals, Economics Letters. 65, (1999), 105-113.

[10] A. Kyle, Continuous Auctions and Insider Trading, Econometrica. 53, (1985), 1315-1335.

[11] H.E Leland, Insider Trading: Should it be Prohibited, Journal of Political Economy, 100, (1992), 859-887.

[12] M. O'Hara, "Market Microstructure Theory", Basil Blackwell, Cambridge, Mass. 1995.

[13] J-C. Rochet and J-L.Vila, Insider Trading without Normality, Review of Economic Studies, 61,(1994), 131-152.

[14] C.E. Tighe,," Three Essays on Insider Trading" unpublished Ph.D dissertation, University of Illinois at Champaign-Urbana, (1989).

CERMSem, Université Paris I, 106-112 Boulevard de l'Hôpital, 75647 Paris Cedex 13, FRANCE; DAHER@UNIV-PARIS1.FR.

Departement of Economics, University of Virginia, Charlottesville, VA 22903, USA; LM8H@CMS.MAIL.VIRGINIA.EDU. 